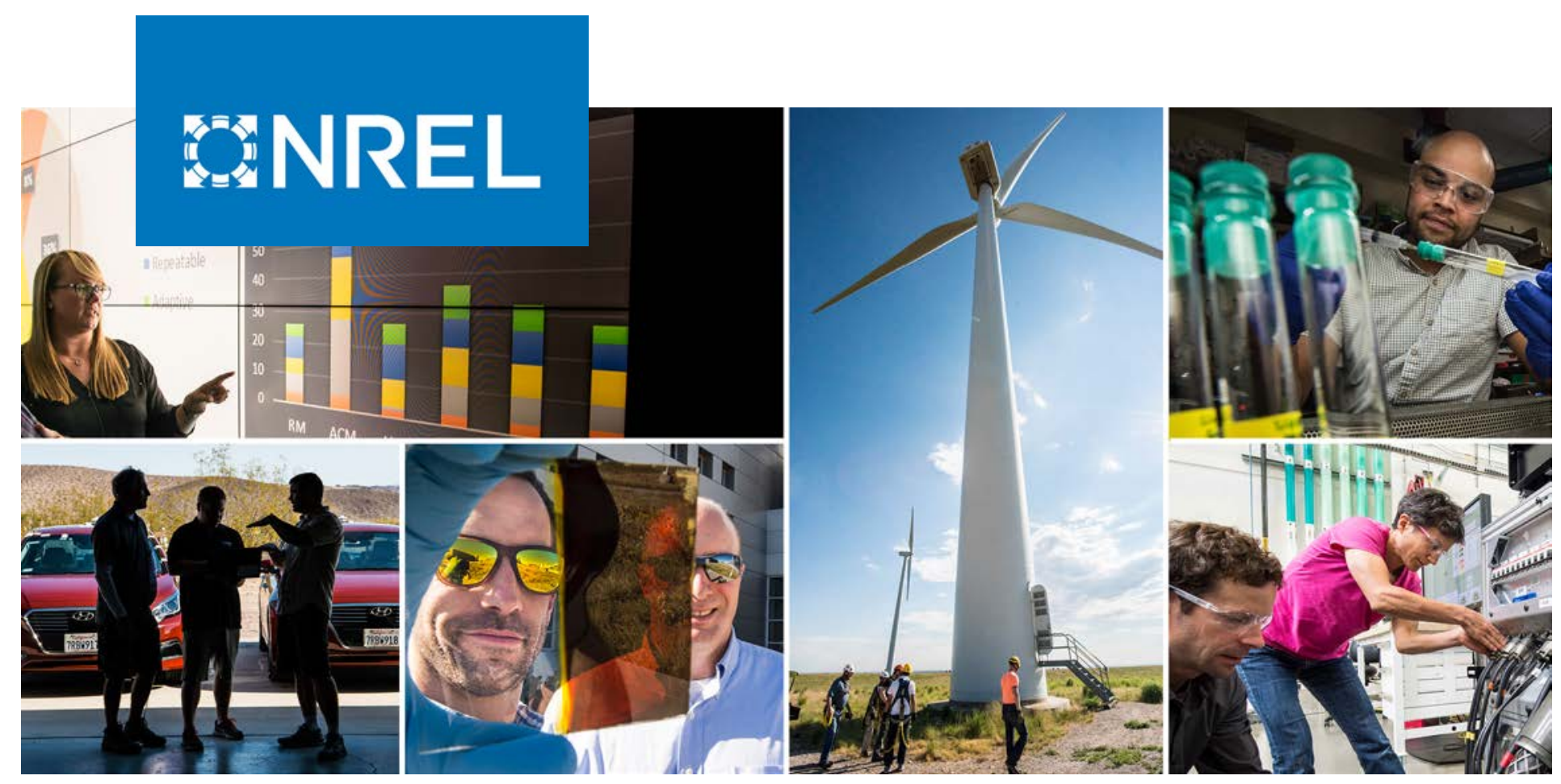

\title{
Multi-Scale Thermal Analysis for Design of SiC-Based Medium Voltage Motor Drive
}

\section{Preprint}

J. Emily Cousineau, ${ }^{1}$ Kevin Bennion, ${ }^{1}$ Karun Potty, ${ }^{2} \mathrm{He} \mathrm{Li},{ }^{2}$ Risha $\mathrm{Na},{ }^{2}$ Longya $\mathrm{Xu},{ }^{2}$ and Jin Wang ${ }^{2}$

1 National Renewable Energy Laboratory

2 The Ohio State University

Presented at ASME 2019 International Technical Conference and Exhibition on Packaging and Integration of Electronic and Photonic Microsystems (IPACK2019) Anaheim, California

October 7-9, 2019

NREL is a national laboratory of the U.S. Department of Energy Office of Energy Efficiency \& Renewable Energy

Operated by the Alliance for Sustainable Energy, LLC

This report is available at no cost from the National Renewable Energy Laboratory (NREL) at www.nrel.gov/publications.

\section{Conference Paper}

NREL/CP-5400-74209

April 2020 


\title{
GNREL
}

\section{Multi-Scale Thermal Analysis for Design of SiC-Based Medium Voltage Motor Drive}

\section{Preprint}

\author{
J. Emily Cousineau, ${ }^{1}$ Kevin Bennion, ${ }^{1}$ Karun Potty, ${ }^{2} \mathrm{He} \mathrm{Li},{ }^{2}$ \\ Risha $\mathrm{Na},{ }^{2}$ Longya $\mathrm{Xu},{ }^{2}$ and Jin Wang ${ }^{2}$ \\ 1 National Renewable Energy Laboratory \\ 2 The Ohio State University
}

\section{Suggested Citation}

Cousineau, J. Emily, Kevin Bennion, Karun Potty, He Li, Risha Na, Longya Xu, and Jin Wang. 2020. Multi-Scale Thermal Analysis for Design of SiC-Based Medium Voltage Motor Drive: Preprint. Golden, CO: National Renewable Energy Laboratory. NREL/CP-

5400-74209. https://www.nrel.gov/docs/fy20osti/74209.pdf

NREL is a national laboratory of the U.S. Department of Energy Office of Energy Efficiency \& Renewable Energy Operated by the Alliance for Sustainable Energy, LLC

This report is available at no cost from the National Renewable Energy Laboratory (NREL) at www.nrel.gov/publications.

Contract No. DE-AC36-08GO28308
Conference Paper

NREL/CP-5400-74209

April 2020

National Renewable Energy Laboratory 15013 Denver West Parkway Golden, CO 80401

303-275-3000 • www.nrel.gov 


\section{NOTICE}

This work was authored in part by the National Renewable Energy Laboratory, operated by Alliance for Sustainable Energy, LLC, for the U.S. Department of Energy (DOE) under Contract No. DE-AC36-08GO28308. Funding was provided by the U.S. Department of Energy Advanced Manufacturing Office. The views expressed herein do not necessarily represent the views of the DOE or the U.S. Government. The U.S. Government retains and the publisher, by accepting the article for publication, acknowledges that the U.S. Government retains a nonexclusive, paid-up, irrevocable, worldwide license to publish or reproduce the published form of this work, or allow others to do so, for U.S. Government purposes.

This report is available at no cost from the National Renewable Energy Laboratory (NREL) at www.nrel.gov/publications.

U.S. Department of Energy (DOE) reports produced after 1991 and a growing number of pre-1991 documents are available free via www.OSTI.gov.

Cover Photos by Dennis Schroeder: (clockwise, left to right) NREL 51934, NREL 45897, NREL 42160, NREL 45891, NREL 48097, NREL 46526.

NREL prints on paper that contains recycled content. 


\section{MULTI-SCALE THERMAL ANALYSIS FOR DESIGN OF SIC-BASED MEDIUM VOLTAGE MOTOR DRIVE}

\author{
J. Emily Cousineau ${ }^{1}$, Kevin Bennion \\ National Renewable Energy Laboratory \\ Golden, Colorado 80401
}

\author{
Karun Potty, He Li, Risha Na, Longya Xu, \\ Jin Wang \\ Department of Electrical and Computer Engineering, \\ The Ohio State University \\ Columbus, Ohio 43210
}

\begin{abstract}
This paper describes a multi-scale thermal analysis approach for the design of an air-cooled 1.7-kV SiC MOSFETbased medium-voltage variable-speed motor drive. The scope of the models and required efficient and flexible thermal models to be developed. Two modeling techniques are described that significantly reduced model run time and enabled more complex models to be run faster while retaining needed accuracy. The first technique uses the effectiveness-NTU method to extract convection boundary conditions from a CFD model that can be applied to a fast-running FEA model. The second is a porous media technique that enables system-level CFD simulations that incorporate effects from heat exchangers (e.g., pin fin heat sinks) that run in a fraction of the time required for fully resolved CFD simulations. The multi-scale approach to the thermal analysis enabled fast and accurate simulation for the converter design ranging from the die level up to the full system with 36 submodules. The modeling results were validated against experimental data from system tests performed by $O S U$.
\end{abstract}

Keywords: Power Electronics, Thermal Modeling, Porous Media, Model Simplification, Effectiveness-NTU

$\begin{array}{ll}\text { NOMENCLATURE } \\ \mathrm{A} & \text { area } \\ \mathrm{C}_{2} & \text { internal resistance factor } \\ \mathrm{CFD} & \text { computational fluid dynamics } \\ \mathrm{CPU} & \text { central processing unit } \\ \mathrm{c}_{\mathrm{p}} & \text { specific heat } \\ \mathrm{FEA} & \text { finite element analysis } \\ \mathrm{h} & \text { convective heat transfer coefficient } \\ \dot{\mathrm{m}} & \text { mass flow rate } \\ \mathrm{MMC} & \text { modular multilevel converter } \\ \mathrm{MOSFET} & \text { metal-oxide semiconductor field-effect } \\ & \text { transistor } \\ \mathrm{NTU} & \text { number of transfer units } \\ \mathrm{p} & \text { pressure } \\ \mathrm{Q} & \text { heat } \\ \mathrm{SiC} & \text { silicon carbide }\end{array}$

$\mathrm{T} \quad$ temperature

UA overall heat transfer coefficient

v velocity

$\begin{array}{ll}\text { Greek Symbols } \\ 1 / \alpha & \text { viscous resistance } \\ \Delta \mathrm{n} & \text { porous media characteristic thickness } \\ \varepsilon & \text { effectiveness } \\ \mu & \text { fluid viscosity } \\ \rho & \text { density }\end{array}$

$\begin{array}{ll}\text { Subscripts } & \\ \mathrm{c}, \mathrm{i} & \text { coolant inlet } \\ \mathrm{c}, \mathrm{o} & \text { coolant outlet } \\ \mathrm{j}-\mathrm{max} & \text { junction maximum } \\ \operatorname{lm} & \text { log-mean } \\ \text { surf,av } & \text { surface average }\end{array}$

\section{INTRODUCTION}

Computer processing power and capability increases continuously, and supercomputers even more so. With these increases come the ability to perform increasingly sophisticated simulations in less time. However, resources, budget, and time are still finite, and will be for the foreseeable future. This paper describes two useful techniques applied to the thermal analysis design of OSU's 1 MVA 1.7-kV SiC MOSFET-based modular multilevel converter (MMC) for driving medium-voltage (i.e., $1 \mathrm{kV}-35 \mathrm{kV}[1])$ electric machines as shown in Figure 1. 


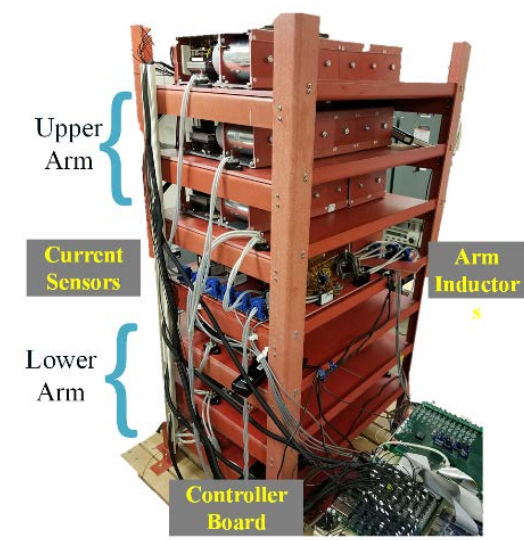

Figure 1: Fully assembled medium-voltage drive converter

Details of the converter design, modeling analysis, and results are discussed in [2]. The tower is about $1.8 \mathrm{~m}$ tall. The drive consists of 36 submodules for three electrical phases. Each submodule is a self-contained unit consisting of an $\mathrm{SiC}$ power module with an air-cooled heat exchanger and capacitor. The middle shelf contains the arm inductors cooled by natural convection. The open rack design and reliance on air cooling and natural convection necessitated running the entire rack in simulation to determine, limit, and eliminate possible hot spots. For the air-cooled system, it was necessary to determine the overall airflow requirements, monitor air temperatures, and support ducting designs for airflow.

The thermal analysis of the system required studying the heat transfer characteristics from the semiconductor device out to the full system level of the complete tower. Using a single model of the full tower system to study the semiconductor module and device temperature profiles would be impractical. To support the thermal analysis and design, the analysis was performed at multiple scales from the chip level up to the full system.

Utilizing the multi-scale approach shown in Figure 2, the problem was divided into interrelated simulations. The modeling approach links a detailed device-scale FEA thermal model of the power module, detailed CFD model of the heat exchanger/fan subsystem, and the system-level full-tower CFD. This work highlights approaches to transfer results between the multiple simulation scales (Figure 1) and the objectives of each model scale.

The FEA model shown in Figure $2 \mathrm{a}$ is a fully resolved thermal model of the power module, including solder layers and attachments. The purpose of the FEA model is to determine the device or MOSFET junction temperatures at specific operating conditions for the converter. The boundary conditions for the model include the device heat loads, convective heat transfer coefficient $(\mathrm{h})$, and ambient air temperature $\left(\mathrm{T}_{\infty}\right)$. The device heat loads were approximated as volumetric heat loads based on the converter operating conditions. Prior to selection of the heat exchanger and fan components, the FEA model was used to identity the initial performance requirements for the heat exchanger. Once a heat exchanger and fan were selected, the convective heat transfer coefficient and ambient temperature were transferred to the heat exchanger/fan CFD model (Figure 2b) and the full tower CFD model (Figure 2c)
The heat exchanger/fan model is a detailed CFD model of the heat exchanger and fan mounted to the power module. The objective of the heat exchanger CFD model is to estimate the convective heat transfer boundary condition (h) applied to the device-level FEA model. In addition, the heat exchanger CFD model provides inputs to the full-tower CFD model, including the heat exchanger pressure drop and the air temperature rise through the heat exchanger. The ambient temperature from the full-tower CFD model feeds into the heat exchanger/fan CFD model as highlighted in Figure $2 \mathrm{~b}$. The convective boundary condition (h) was estimated utilizing the effectiveness-NTU heat exchanger properties extracted from the fully resolved heat exchanger model (Figure 2b) and ambient temperature determined from the heat exchanger CFD model and full tower model (Figure 2c).

The full-tower CFD model (Figure 2c) focused on simulating the airflow through the entire system. The objective of the full-tower CFD model was to ensure uniform airflow and temperatures through the submodule assemblies. The model extracted the inlet air temperature to each of the submodules, which were used in the previously described module FEA thermal model and heat exchanger CFD model. The pressure drop calculated from the heat exchanger CFD model (Figure 2b) was used to construct an equivalent porous media region that was used to reduce computational requirements for the fulltower CFD model shown in Figure 2c.

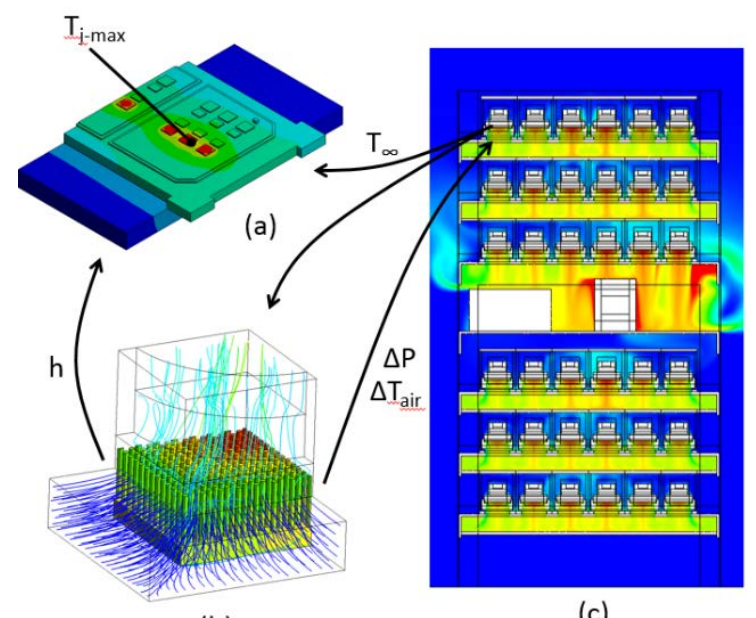

(b)

(c)

Figure 2: The three primary models used in analysis and their interrelationships. The arrows denote properties that were obtained from each model as inputs to the other models. (a) Device-scale FEA model. (b) Heat exchanger CFD model. (c) Full-tower CFD model.

The benefits of the technique are clear. The quartersymmetry heat exchanger shown in Figure $2 \mathrm{~b}$ consisted of 5.2 million elements and took about 16 CPU-hours to solve. The FEA model shown in Figure 2a could only take advantage of one symmetry plane; thus, if a fully resolved model of the heat exchanger was used, it would require doubling the number of required control volumes and hence time to solve. However, extracting a convection coefficient from the quarter symmetry and applying it to the FEA in lieu of a fully resolved model resulted in a model that gave the same results, but with substantially reduced computation time. On the other end, using 
a porous media simplification in place of the pin-fin heat exchanger facilitated running full-scale CFD simulations of the MMC tower. The scope of the simulation domain in the full MMC tower model was such that it would not have been possible to run within the time constraints of the project without using the porous media simplification.

This paper highlights the techniques used for linking the models. Two model simplifications, effectiveness-NTU and porous media, will be described. Both simplifications provide substantial resource and time savings with minimal sacrifice in accuracy when applied appropriately. The procedure for each model simplification will be shown in detail, as well as the results and limitations of each approach.

\section{APPROACH}

This paper highlights the approach to analytically link the different model scales to efficiently model the system from the device level to the full tower. Figure 3 summarizes the different simulation scales and highlights what is transferred between the modeling scales. The effectiveness-NTU method links the device and power module FEA model to the heat exchanger CFD. The porous media model links the heat exchanger CFD to the submodule and full-tower CFD. Each of these methods is described in more detail below.

Figure 3 shows the different simulation scales used in the project. In addition to the simulation scales highlighted in Figure 2, an additional "debugging" simulation scale (Submodule CFD in Figure 3) was added to minimize the number of times the full system model needed to be computed.

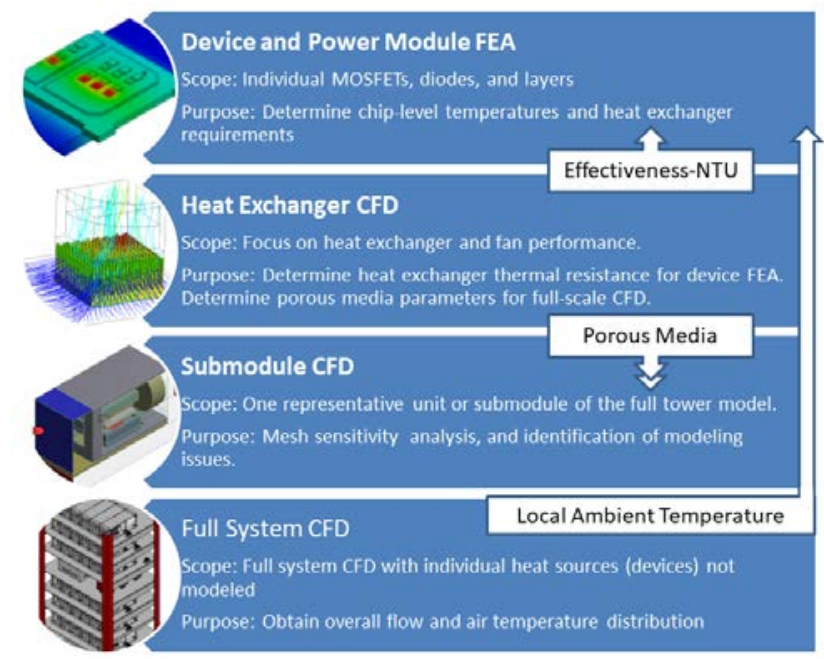

Figure 3: Simulation scales and relationships utilized in $\mathrm{MMC}$ analysis.

\subsection{Effectiveness-NTU Technique}

The effectiveness-NTU method was chosen to represent the characteristic convective heat transfer coefficient of the heat exchanger. The approach was chosen because it captures the effect of the fluid properties and flow rate within the heat exchanger as highlighted by Moffat [3]. The approach described below outlines a method of extracting the effective convective heat transfer coefficient from a CFD model using the effectiveness-NTU approach. The benefit of the approach is it allows the performance and the heat exchanger to be modeled within the FEA thermal model with a convective heat transfer boundary condition.

A schematic of the effectiveness-NTU technique is shown in Figure 4. Note that the heat exchanger's heat spreader is included in both the FEA and CFD models. The technique takes advantage of the physical purpose of the heat spreader to average the heat flow from the baseplate into the heat exchanger fins.

The first step in extracting an effective convective heat transfer coefficient from the heat exchanger CFD model was to extract the average heat flux. For simple setups where all of the heat generated by the package is dissipated by a single heat spreader, this calculation is trivial (total heat divided by total contact area between the module base plate and heat spreader). If multiple heat spreaders are used, an initial FEA may be required to obtain the appropriate heat fluxes for each heat exchanger.

Extraction of the convection coefficient is an analytical process outlined in the literature [3], [4]. The process is stepped through below for convenience to the reader. The calculation begins with obtaining the differences in temperatures between the surface of the heat spreader, and inlet and outlet temperatures $(\Delta \mathrm{T})$ as shown in Equation 1.

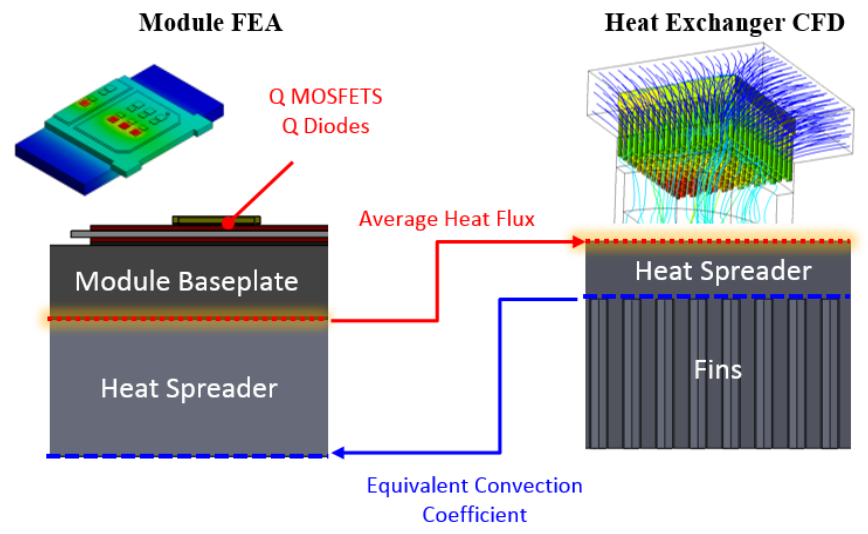

Figure 4: Schematic of effectiveness-NTU technique.

$$
\Delta T_{1}=T_{\text {surf }, a v}-T_{c, o} ; \Delta T_{2}=T_{\text {surf }, a v}-T_{c, i}
$$

where $T_{\text {surf,av }}$ is the area average surface temperature of the plane where the heat spreader meets the fins, including the surface temperature and the cross-section through the fins. Tc,o and Tc,i are the mass flow average temperatures of the coolant at the outlet and inlet, respectively. Overall heat transfer coefficient (UA) uses the log-mean temperature difference as calculated in Equation 2.

$$
\Delta T_{l m}=\frac{\left(\Delta T_{1}-\Delta T_{2}\right)}{\log _{e}\left(\Delta T_{1} / \Delta T_{2}\right)}
$$

Next, the UA is obtained in Equation 3.

$$
U A=Q / \Delta T_{l m}
$$


where $\mathrm{Q}$ is total heat through the control surface. In our experience, 1/UA tends to overestimate the convection coefficient when using Tc,i, so the NTU refinement is used as shown in Equation 4.

$$
N T U=U A / \dot{m} c_{p}
$$

where $\dot{m}$ is the mass flow through the system, and $c_{p}$ is the mass flow average specific heat of the fluid volume. Typically, a heat exchanger will have a high enough flow rate that the specific heat does not change significantly as it passes through the heat exchanger. If the specific heat changes substantially (such as a phase change), this technique will not be valid. Finally, the effectiveness $\varepsilon$ is calculated as shown in Equation 5.

$$
\varepsilon=1-e^{-N T U}
$$

The convection coefficient (h) is calculated in Equation 6 and applied to the FEA model as shown in Figure 4.

$$
h=\varepsilon \dot{m} c_{p} / A
$$

where A is the contact area between the module baseplate and heat exchanger spreader.

\subsection{Porous Media Approximation}

It is often impractical to geometrically model every detail of a component in a computational model; therefore, simplifications are used. For example, electric machines have multiple composite components (e.g., windings, lamination stacks). For an FEA thermal model, modeling each individual wire within a winding or each sheet of metal within a lamination stack would be extremely time consuming. Instead these composite components are modeled with representative thermal properties that treat the component as a continuum to reach a solution [5]. There is also an analogue of the composite material for fluid flow in CFD called porous media. Instead of resolving the flow field through intricate passages and fins of a heat exchanger, a zone with effective pressure losses can be substituted to simplify the model while still retaining the intent. The porous media model requires two inputs: viscous resistance and internal resistance factors. While there are multiple methods to calculate these factors, this paper will cover a simple method that uses pressure and velocity data from the fully resolved model [6].

The first step is to obtain a quadratic equation relating pressure drop $(\Delta \mathrm{p})$ to velocity $(\mathrm{v})$ as shown in Equation 7 . In this case, the air velocity range was used to represent the air velocities obtained in the heat exchanger-level CFD. Simulated data are not subject to noise or measurement uncertainty, so only a minimum number of points are needed to obtain the coefficients for Equation 7. If these were actual experimental data, it would be advisable to obtain at least three times as many data points. If the data do not follow the quadratic curve in Equation 7, it may be an indication that the porous media approximation is unsuitable for the system. Note that the coefficient $\mathrm{C}$ is calculated in Equation 7. Physically, $\mathrm{C}$ should be zero; however, it was found that forcing the curve fit through the origin produced less agreement between the fully resolved and porous media models than a curve that is allowed to offset.
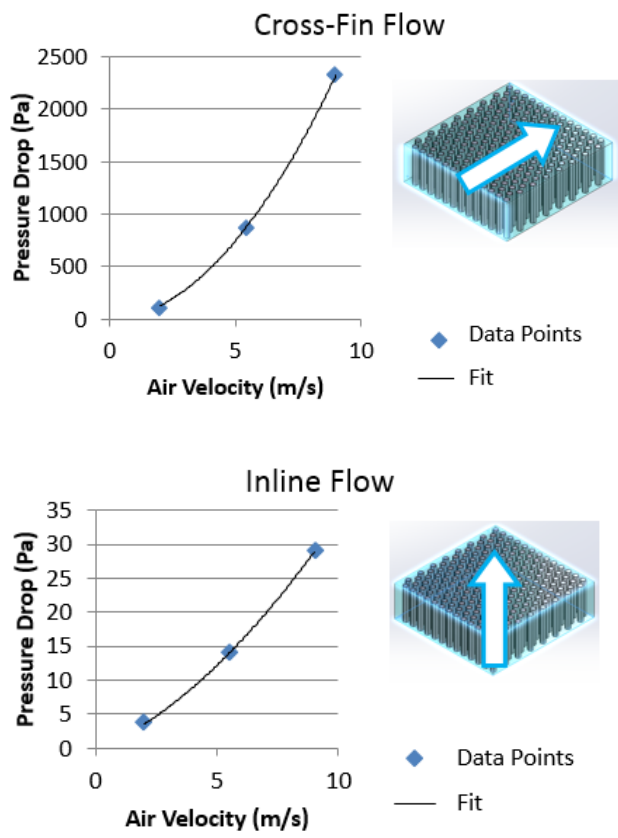

Figure 5: Pressure drop versus velocity plots for cross-fin (top) and inline flow (bottom). The flow direction relative to the fins is shown at the right of each plot.

Because the pressure drop is different for flow across the fins as compared to flow in-line with the fins, it was necessary to run simulations for both flow directions of the cross-fin flow shown in Figure 5. The resulting porous zone was orthotropic.

$$
\Delta p=A v^{2}+B v+C
$$

From Equation 7, the viscous resistance $(1 / \alpha)$ and the internal resistance factor $\left(\mathrm{C}_{2}\right)$ can be obtained using the relationships shown in Equations 8 and 9.

$$
1 / \alpha=B /(\mu \cdot \Delta n)
$$

where B is the coefficient from Equation 7, $\mu$ is the viscosity, and $\Delta \mathrm{n}$ is the thickness of the porous media region in line with the flow.

$$
C_{2}=2 A /(\rho \cdot \Delta n)
$$

where A is the coefficient from Equation 7, and $\rho$ is the density of the fluid.

Once the above procedure was applied to develop the porous media parameters specific to the pin fin heat sink, it was necessary to correctly determine the heat transfer from the solid zone to the fluid zone. For this it was necessary to calculate the porosity. For this model, the porosity was determined to be 0.96 using a simple Newton-Raphson iterative process to find the porosity value that resulted in the same temperature rise of the fluid as the fully resolved pin fin model.

Finally, the porosity parameter (in ANSYS Fluent) was used to tune the thermal performance of the porous media heat exchanger. 


\section{RESULTS \& DISCUSSION}

The effectiveness-NTU and porous media methods provided significant time saving benefits compared to the fully resolved models, with only a modest reduction in fidelity. The reduction in model fidelity was far outweighed by the throughput and responsiveness of the simplified models. The effectiveness-NTU technique resulted in a tremendously faster solution speed than the fully resolved heat exchanger model. Key results, such as maximum junction temperature, matched the fully resolved model exactly. The porous media approximation resulted in a $90 \%$ reduction in run-time and memory usage for the model, with only a modest reduction in accuracy.

To validate the model, a negative temperature coefficient (NTC) thermistor was included in the FEA device model analogous to the NTC on the physical power module. The temperature measurement of the $\mathrm{NTC}$ was $110^{\circ} \mathrm{C}$, and the FEA computed value for the same location was $106^{\circ} \mathrm{C}$. The $4^{\circ} \mathrm{C}$ difference between model and experiment was considered acceptable given the temperature limit of $125^{\circ} \mathrm{C}$.

\subsection{Effectiveness-NTU Technique Results}

As mentioned above, the device FEA model using a convection coefficient calculated using the effectiveness-NTU technique gave identical results to the fully resolved CFD heat exchanger model, which are shown in Figure 6. The notable difference was the time to reach the solution. The fully resolved model took $16 \mathrm{CPU}$-hours to solve, amounting to about 15 minutes to obtain a solution on a 64-core system. However, a solution could be obtained from the device FEA model in just under 3 seconds on a standard workstation. This resulted in a significant amount of flexibility and design space exploration that would not have been possible with a fully resolved model. It also enabled transient simulations to be run which would not have been possible to achieve within the budget and time constraints of the project using the fully resolved model.

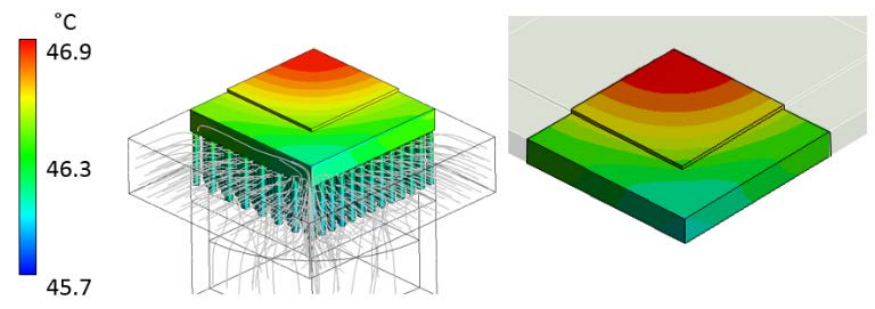

Figure 6: Comparison of surface temperature of plate heater mounted on heat exchanger. Left: Fully resolved CFD model. Right: FEA model with effective convection coefficient.

The intrinsic limitation of the device FEA model was that it could not predict temperature rise in the fluid or evaluate different fluid flow rates, which require running the fully resolved CFD heat exchanger model and recalculating the convection coefficient, as necessary. Another limitation would be if the temperature distribution in the heat exchanger varied significantly enough to result in variation in local heat transfer coefficients. If the fluid flow rate was so slow that the properties could change significantly as the fluid moved through the heat exchanger, the technique may not be valid. General quantification of when the effectiveness-NTU simplification breaks down is beyond the scope of this paper. It is always a good practice to verify simplifications against high-fidelity data.

\subsection{Porous Media Results}

The differences in key parameters are shown in Table 1. Average refers to mass flow average. Fan velocity is the average velocity through the fan zone. $\Delta \mathrm{P}$ is the average pressure drop from the inlet to the fan boundary. $\Delta \mathrm{T}$ ave is the average temperature rise through the porous zone. $\Delta \mathrm{T}$ max is the maximum temperature rise through the porous zone, which was matched to the fully resolved model by adjusting the porosity parameter in ANSYS Fluent.

Table 1: Comparison of critical parameters between the fully resolved model and porous media model.

\begin{tabular}{lcccc} 
& $\begin{array}{c}\text { Fan Velocity } \\
(\mathrm{m} / \mathrm{s})\end{array}$ & $\begin{array}{c}\Delta \mathrm{P} \\
(\mathrm{Pa})\end{array}$ & $\begin{array}{c}\Delta \mathrm{T} \\
\text { average } \\
\left({ }^{\circ} \mathrm{C}\right)\end{array}$ & $\begin{array}{c}\Delta \mathrm{T} \\
\max \end{array}$ \\
\hline$\left.{ }^{\circ} \mathrm{C}\right)$
\end{tabular}

With the exception of the average velocity through the fan boundary, the critical parameters were within $2 \%$ of the fully resolved model. The porous media model differed from the fully resolved model by $13.5 \%$ for the average velocity through the fan zone. A notion as to why this occurred is shown in Figure 7.

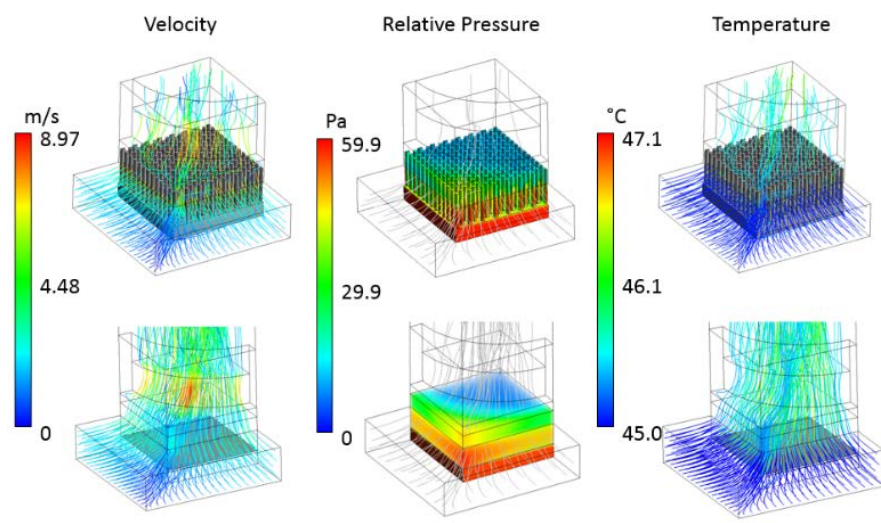

Figure 7: Comparison of critical parameters between the fully resolved model (top) and porous zone model (bottom).

The porous zone accurately replicates the pressure and temperature fields through the heat exchanger. The velocity field was not accurately captured because the porous media approximation only models the pressure drop through the heat exchanger. In the fully resolved model, the geometry causes constrictions through which the flow must pass, resulting in higher localized velocities, boundary effects, and changes in direction as the air flows past the obstacles. No such obstacles exist in the porous zone. This is an important limitation of the porous media model. It cannot replicate the flow field through the zone. Fortunately, resolving the velocity field through the 
heat exchangers was not necessary in the full system model, only the fluid temperature rise and pressure drops through the heat exchangers were needed.

Accepting the $2 \%$ reduction in accuracy of pressure drop and temperature rise in the porous media model reduced the run time of the full system model dramatically. While a version of the full system model containing fully resolved heat exchangers was not run, it was estimated based on the number of control volumes saved by using the porous media model that the run time was reduced by $83 \%$. This translated into a time savings of 108,000 CPU-hours on hardware available at the time of the project (2017). Put another way, on a 128-core system a fully resolved full-system model would have taken six weeks to complete and required prodigious quantities of physical memory, while the full system model using the porous media model only took about seven days to run.
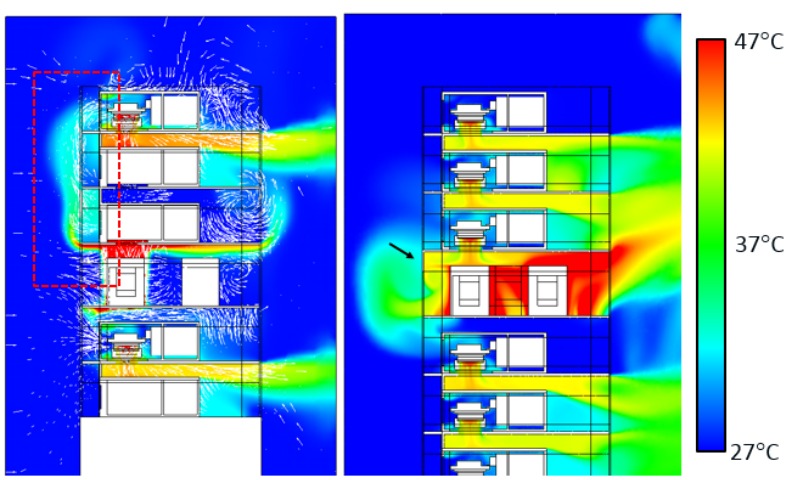

Figure 8: Left, the simulation revealed an air flow issue that was corrected easily with a baffle (right). The left image only has a few submodules included to reduce time requirements, while the right image is representative of a final run including all submodules used as a final check of the system.

The full system model was able to identify issues with airflow for the system. For example, in Figure 8 it was identified that heat coming off an inductor on the fourth shelf would create a plume that ended up being pulled into a module on the top shelf, raising its temperature by several degrees. By installing a simple baffle on the front of the shelf above the inductor bank the issue was resolved. Utilizing the porous media simplification enabled the full system model to be run multiple times, allowing identification and correction of flow issues prior to turning on the MMC tower experimental setup, and potentially saving the expense of having to identify the problem and, in the worst-case, replacing a failed module. The issue would have been difficult to detect in the actual test setup due to safety requirements (no one was allowed in the room while operating) and would have only showed up as a hot-spot that would have resulted in project delays to identify and resolve.

\section{CONCLUSION}

This paper outlines two methods for simplifying computational models to significantly reduce run time and resource requirements, while obtaining reliable and accurate results. The effectiveness-NTU method is used to generate boundary conditions for a detailed, fast-running FEA model from a fully resolved CFD model. The effectiveness-NTU method allows engineers to simultaneously take advantage of the fidelity of CFD simulation and the speed of FEA simulation, reducing the time requirements for analyzing "what-if" scenarios and design changes. The porous media method enables analysis of flow fields around heat exchangers without the additional computational expense of geometrically modeling the heat exchanger itself, facilitating system-level simulation. By performing system-level simulations it was possible to evaluate the entire design and check solutions to potential problems before running the experimental setup, reducing risk of failed parts and improving safety.

\section{ACKNOWLEDGMENTS}

This work was authored in part by the National Renewable Energy Laboratory, operated by Alliance for Sustainable Energy, LLC, for the U.S. Department of Energy (DOE) under Contract No. DE-AC36-08GO28308. Funding was provided by the U.S. Department of Energy Advanced Manufacturing Office. The views expressed in the article do not necessarily represent the views of the DOE or the U.S. Government. The U.S. Government retains and the publisher, by accepting the article for publication, acknowledges that the U.S. Government retains a nonexclusive, paid-up, irrevocable, worldwide license to publish or reproduce the published form of this work, or allow others to do so, for U.S. Government purposes.

\section{REFERENCES}

[1] "IEEE Guide for the Functional Specification of Medium Voltage (1 kV-35 kV) Electronic Shunt Devices for Dynamic Voltage Compensation," IEEE Std 1623-2004, pp. 0_1-17, 2005.

[2] H. Li et al., "Hardware Design of A 1.7 Kv Sic MOSFET Based MMC For Medium Voltage Motor Drives," 2018, pp. 1649-1655.

[3] R. J. Moffat, "Modeling Air-Cooled Heat Sinks as Heat Exchangers," in Twenty Third Annual IEEE

Semiconductor Thermal Measurement and Management Symposium (SEMI-THERM), San Jose, CA, 2007, pp. 200-207.

[4] F. P. Incropera, D. P. DeWitt, T. L. Bergman, and A. S. Lavine, Eds., Principles of Heat and Mass Transfer, 7. ed., international student version. Singapore: Wiley, 2013.

[5] D. Staton, A. Boglietti, and A. Cavagnino, "Solving the More Difficult Aspects of Electric Motor Thermal Analysis in Small and Medium Size Industrial Induction Motors," IEEE Trans. Energy Convers., vol. 20, no. 3, pp. 620-628, Sep. 2005.

[6] "Deriving the Porous Coefficients Based on Experimental Pressure and Velocity Data.” ANSYS Help Manual 16.2 Section 6.2.3.6.11. 